\title{
The new Avicenna Roboflex: How does the irrigation system work? Results from an in vitro experiment
}

\author{
Salvatore Butticè ${ }^{1,2,6}$, Bahadir Sahin ${ }^{3}$, Tarik Emre Sener ${ }^{3,6}$, Laurian Dragos ${ }^{4,6}$, Silvia Proietti ${ }^{5,6}$, \\ Steeve Doizi ${ }^{1,6}$, Olivier Traxer ${ }^{1,6}$ \\ ${ }^{1}$ Pierre \& Marie Curie University, Tenon University Hospital, Paris, France; \\ ${ }^{2}$ Department of Urology, San Giovanni di Dio Hospital, Agrigento, Italy; \\ ${ }^{3}$ Department of Urology, School of Medicine, Marmara University, Istanbul, Turkey; \\ ${ }^{4}$ Department of Urology, Emergency County Hospital, Pius Branzeu, Timisoara, Romania; \\ ${ }^{5}$ Ville Turro Division, Department of Urology, IRCCS, Ospedale San Raffaele, Milan, Italy; \\ ${ }^{6}$ Members of PETRA UroGroup, Progress in Endourology, Technology and Research Association.
}

\begin{abstract}
Summary Introduction: Since 2012 Elmed has been working on a robot specifically designed for flexible ureteroscopy. After the first version of Avicenna Roboflex, a second version was developed in 2015, with signifcant changes especially in the irrigation system. We consider mandatory for the endourologist that works with the Avicenna Roboflex be aware of the functioning of the irrigation system. Materials and Methods: We connected a container to the pump's irrigation system and measured the quantity of saline per second delivered by each speed setting, with/without the flush in five different modalities: pump on its own, pump with ureteroscope, with two laser fibers, with $1.9 \mathrm{Fr}$ basket, and with a Terumo guidewire.

Results: The highest mean flow-rates were observed in the 200micrometer laser fiber, after the pump on its own. Median flowrates for all speed settings were significantly higher for the pump on its own than for the URS in both flushed and nonflushed modes ( $p=0.045, p=0.039$ respectively). There was no statistically significant difference in median flow-rates between the guide wire and basket in all of the speed settings ( $p=0.932$ and $p=0.977$ ). For both laser fibers there was no statistically significant difference between the median flow rate on both nonflush and flush modes. $(p=0.590 \& p=0.590)$. There was a linear correlation between the speed setting and the increase measured with the flush-option for pump only measurements $(r=0.602, p=0.038)$. There was no statistically significant difference between laser fibers and the pump on its own on the increase of flow rate with flush mode. ( $p=0.443$ for the 272 micrometer fiber and $p=0.219$ for the 200-micrometer fiber). Conclusion: The irrigation system of the new Avicenna Roboflex is optimized compared to the previous version. However other more complex studies concerning the live flow/pressure relationship are needed before firm conclusions can be made.
\end{abstract}

KEY WORDS: Renal stone; Ureteroscopy; Avicenna Roboflex; Irrigation system; Flexible ureteroscopy; Intrarenal flow; Urolithiasis.

Submitted 24 March 2018; Accepted 29 April 2018

\section{INTRODUCTION}

Urolithiasis is one of the major issues in healthcare, with an incidence of around 10\%, while the use of flexible ureteroscopes has increased exponentially as one of the best treatment options for renal stones (1).
The rapidly growing popularity of flexible ureteroscopy (FURS) has also been sustained by the major companies on the market, which have increased efforts to develop flexible ureteroscopes. Indeed no other endourological device has received more attention or undergone more dynamic changes than these delicate endoscopes (2). Thus, adaptation of FURS to robotic surgery has been inevitable with all the advancements in technology. In fact, Elmed (Ankara, Turkey) has been working on a robot specifically designed for FURS since 2012 (3).

After the first version of Avicenna Roboflex in 2012, a second version was developed in 2015, with changes to the console, improved ergonomics and with significant changes in the irrigation system. Today the mechanics and electronics of Roboflex has been experienced and published but the precise flow produced by the pump in each of the different speed settings remains somewhat unexplored (4).

In fact, the first version provided 25 different speed options for the irrigation system, whereas the second one provides 12 speeds, with a dramatic change in the dynamics of intra-renal flow (5). For this reasons we consider it mandatory that the endourologists who work with the new Avicenna Roboflex be aware of the pump flow rate for each speed setting.

\section{The irrigation system}

As mentioned above, the irrigation system is controlled by a 12-speed mechanical pump and can be attached to a regular rod for gravitational irrigation. The entire device is powered electronically and has two small rotors in the front part to which an infusion tube is connected; which is compatible with others on the market or with the included piece (Figure 1) The system is connected to a console with four buttons: one to start and stop, one to increase and another to decrease the flow, and the flush (Figure 2) The "flush" allows a rapid increase in flow for about one second, and is different from other mechanical systems that permit a saline adjustment; the flush can be operated approximately every 2 seconds after it has been activated; a refractory time that varies from 1.5 to 2 seconds by switching from low to high speed. 


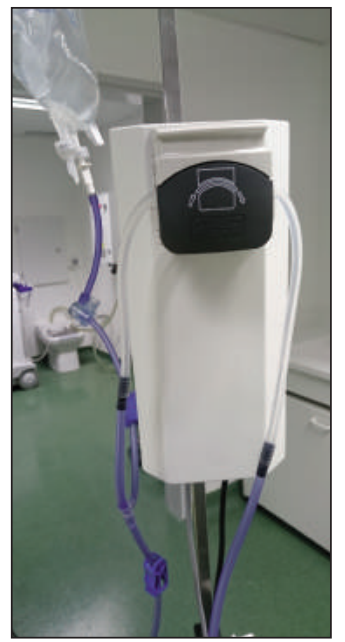

Figure 1.

The pump of the irrigation system of the new Avicenna Roboflex.

Figure 2.

The console of Avicenna Roboflex, it is possible to note on the bottom right, the part that controls the entire irrigation system electronically.

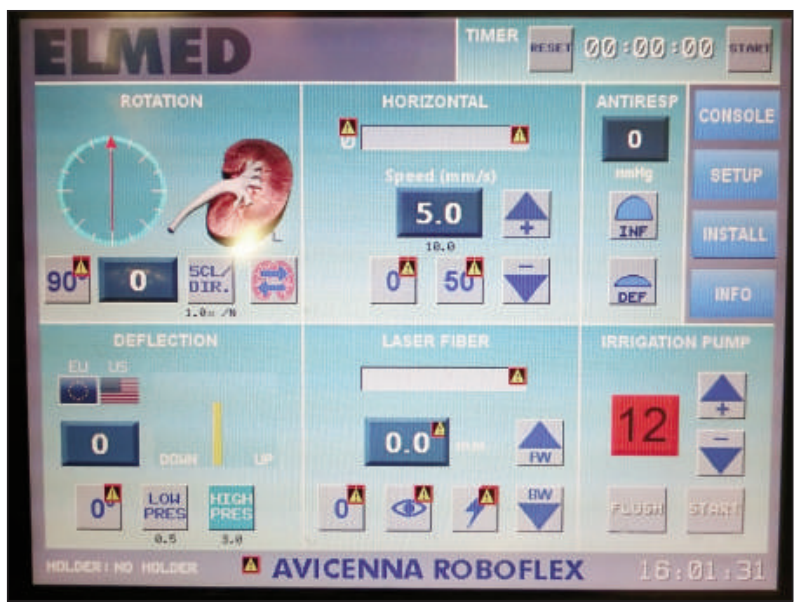

\section{Materials AND Methods}

This is an in vitro study in accordance with the Helsinki Declaration, conforms to the Committee on Publication Ethics (COPE) guidelines, and was approved by the Institutional Review Board (IRB) of the University Hospitals in which the study was carried out. The design, analysis, interpretation of data, drafting, and revisions of the study followed the Strengthening the Reporting of Observational Studies in Epidemiology (STROBE) Statement as well as the guidelines for reporting observational studies, available through the Enhancing the Quality and Transparency of Health Research (EQUATOR) network (6).

For the study, we connected a container to the pump's irrigation system and measured the quantity of saline solution per second delivered by each speed setting, both with and without the flush (for 30 seconds for every measurement) in five different modalities: the pump on its own, the pump with ureteroscope (Olympus URF-P5), with two laser fibers (200 and $270 \mu \mathrm{m}$ by Rocamed), with a 1.9 Fr basket (Dakota by Boston Scientific), and with a 0.018 inch Terumo guidewire attached. The pump was fixed to a rod that is used for regular urological irrigation, at a height of 1.60 meters. As infusion tube, we used a T-flow by Rocamed which permits the addition of a manual stream via an integrated pump but the pump was not used in this case. Every measurement was performed 5 times and the mean values were taken for each modality and speed setting (Figure 3).

Figure 3.

Setting of the experiment at Tenon Hospital.

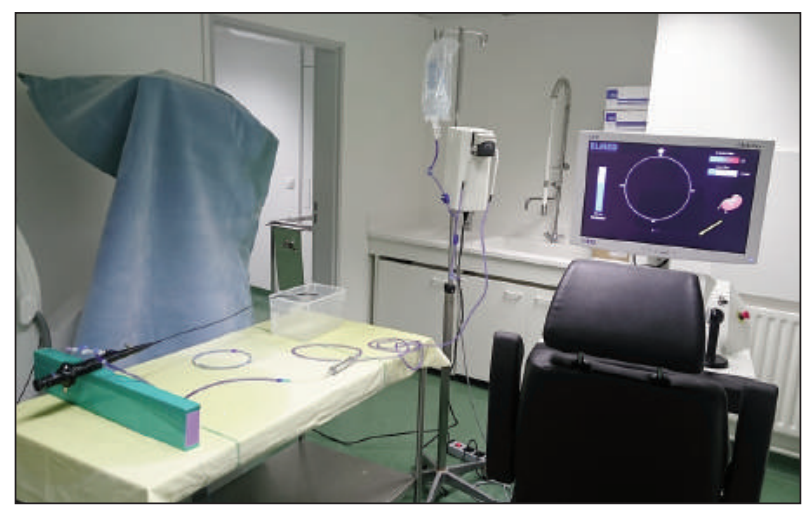

\section{Statistical analysis}

Statistical analyses were performed using the original SPSS software, version 22.0 (IBM Corp, NY, USA), with significance set at $p<0.05$. Baseline variables were described using means and standard deviations, or medians and minimum, maximum values as appropriate. Mann-Whitney U tests were used to evaluate the difference between quantitative measurements that have non-parametric distribution. Linear correlation between numeric variables that have a non-parametric distribution were evaluated with spearman rank coefficient. Sensitivities, specificities, and predictive values with $95 \%$ confidence intervals were calculated.

\section{RESULTS}

The measured mean flow-rate values for different modalities (sets of instruments) with and without flush-mode are given in Table 1. The measured values indicate that the highest mean flow-rates were observed in the 200micrometer laser fiber, after the pump on its own.

The flow-rates for the 200-micrometer laser fiber were lower in the first four speed-settings than the flow-rates of the ureteroscope (URS). However, after the fourth speedsetting, faster flow rates were measured with the 200micrometer laser fiber. The same pattern applied to the 272-micrometer laser fiber except that faster flow-rates

Table 1.

Mean flow rates.

\begin{tabular}{|lcc|}
\hline & Without flush & With flush \\
\hline Free pump & 52 & 54.9 \\
\hline URS & 33.9 & 34.8 \\
\hline Guidewire & 7.8 & 8.1 \\
\hline Basket & 7.1 & 7.7 \\
\hline $\mathbf{2 7 2} \mathbf{~ m m}$ Laser fiber & 38 & 39.7 \\
\hline $\mathbf{2 0 0} \mathbf{~ m m}$ Laser fiber & 41.2 & 42.8 \\
\hline
\end{tabular}


were achieved after the fifth setting instead of the fourth. Median flow-rates for all speed settings were significantly higher for the pump on its own than for the URS in both flushed and non-flushed modes ( $p=0.045, \mathrm{p}=$ 0.039 respectively). There was a $33.97 \%$ drop in flowrate for the non-flush mode of the URS compared to the non-flush mode of the pump on its own.

There was no statistically significant difference in median flow-rates between the guide wire and basket in all of the speed settings, although the median flow-rate with the guide wire was slightly higher in both non-flush and flush modes $(\mathrm{p}=0.932$ and $\mathrm{p}=0.977$ ).

The mean decrease during non-flush mode with the guide wire was $87.2 \%$ and $81 \%$ compared to the pump on its own and the URS respectively. For the basket these drop rates were $88 \%$ and $82.1 \%$. Instead, the median rates of increase during flush mode were 0.20 (0.10$1.20)$ and $0.30(0.10-4.00) \mathrm{ml} / \mathrm{min}$ for the guide wire and the basket respectively. There was no statistically significant difference between the mean increase in flowrates for flush mode between the basket and the guide wire options $(p=0.378)$. For both laser fibers there was no statistically significant difference between mean flowrates in all of the speed settings, although the mean flow rate for the 200-micrometer fiber was markedly higher ( $\mathrm{p}=0.590 \& \mathrm{p}=0.590)$. The mean decreases in nonflush mode were $32.9 \%$ and $27.2 \%$ compared to the pump on its own for the 272-micrometer and the 200micrometer laser fibers respectively. The decrease in rate compared to the pump on its own was inversely related to the speed setting for both laser fiber groups (Table 2).

\section{Table 2.}

Correlation between increase with flush mode and increase in speed setting.

\begin{tabular}{|lll|}
\hline Free pump & $\mathbf{r}$ & $\mathbf{p}$ \\
\hline URS & 0.602 & 0.038 \\
\hline Guidewire & 0.339 & 0.282 \\
\hline Basket & 0.562 & 0.057 \\
\hline $\mathbf{2 7 2} \mathbf{~ m m}$ Laser fiber & 0.504 & 0.095 \\
\hline $\mathbf{2 0 0} \mathbf{~ m m}$ Laser fiber & 0.644 & 0.024 \\
\hline$r:$ Spearman correlation coefficient. & 0.329 & 0.208 \\
\hline
\end{tabular}

There was a linear correlation between the speed setting and the increase measured with the flush-option for pump only measurements $(r=0.602, p=0.038)$.

The same correlation was also observed for the 272 micrometer laser fiber $(p=0.664, p=0.024)$ but not for the other disposables (Figure 4).

With the use of the guide wire, basket, or URS, the median rates of increase for the flush mode were respectively $0.20(0.10-1.20), 0.30(0.10-4.00), 0.90$ (0.4-1.30) $\mathrm{ml} / \mathrm{min}$. These values were significantly lower than the pump on its own which was $1.70 \mathrm{ml} / \mathrm{min}(0.80-11.90)$ ( $p<0.001$ for all three). For both laser fibers, rates of increase for flush mode were respectively 1.50 (0.40$3.80)$ and $1.35(0.60-3.70) \mathrm{ml} / \mathrm{min}$. There was no statistically significant difference between laser fibers and the pump on its own ( $p=0.443$ for the 272-micrometer fiber and $\mathrm{p}=0.219$ for the 200-micrometer fiber).

\section{Discussion}

The irrigation system is a fundamental component used during FURS because it improves visualization, maintains patency of the urinary tract. Besides, pressurized irrigation is necessary to maintain sufficient distension of the lumen when accessory instruments (baskets, laser fibers, etc.) are passed through a small working channel (2). In a recent study it had been shown that with the use of 273-micrometer laser fiber flow volume decreases 53.7\%. Although different sets of laser fibers are used in our study it has been showed that this new irrigation system causes minimal or no loss on flow rate with laser fibers depending on the speed setting preferred by the surgeon (7).

The mechanical irrigation systems that are currently on the market provide a continuous flow that can be integrated manually. This is the first pump on the market that allows for robotic adjustment of the flow whilst increasing it according to the needs of the endourologist. When comparing the new irrigation system to the older one, it can immediately be seen that the new pump has a more constant flow when switching to a higher speed. Though the "old pump" had more than 25 speed settings, it had a difference in flow rate of $8-10 \mathrm{ml} / \mathrm{min}$ at intermediate speed settings, which could potentially develop dangerously high intrarenal pressures. For these reasons, in our previous work we advised caution using the pump
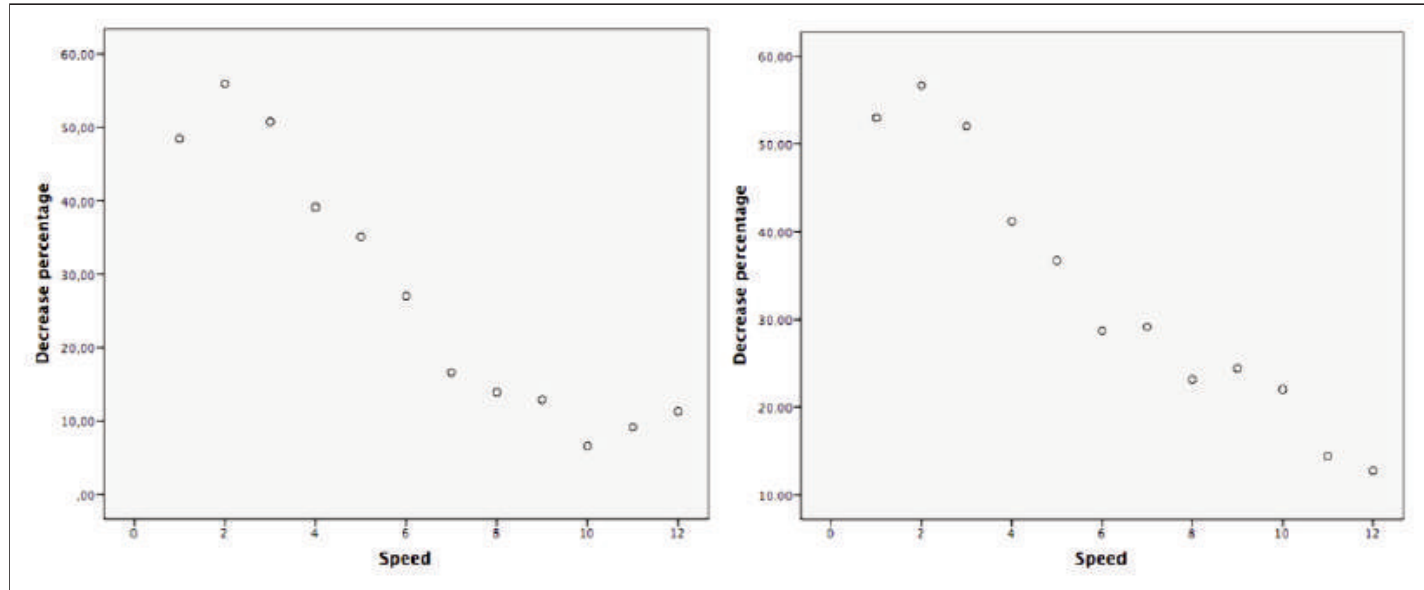

Figure 4.

Decrease of flow rate for $272 \mathrm{~mm}$ laser fiber and 200 $\mathrm{mm}$ laser fiber compared to free pump $(r=-0.979$, $p=<0.001$ $\& r=-0.951$, $p=<0.001)$ 
at intermediate speed settings (5). Although this study does not assess intrarenal pressures, a profile of a safe range of kidney pressure should be developed. The only measurement of pressure using the Avicenna Roboflex was that done by Rassweiler J et al. at WCE 2015 who used an intra-pelvic sensor and reached a maximum pressure of 40 $\mathrm{cm}_{2} \mathrm{O}$ (8). However, this pump has an important limitation; during typical FURS the endourologist or assistant who injects saline by manual pump has a pressure feedback, and is able to sense when intrarenal pressure rises. This depends on subjective feeling and is not scientifically comparable to real intrarenal pressure, but since most centers still do not have a measurement system of renal pressure, the "tactile" sense remains the only means available to the urologist. However, even with feedback on hand, when the operator needs an additional stream and does not have an automated system, they cannot know the quantity of fluid or how fast they are injecting it, into the renal cavity. This situation may result in dangerously unrecognized high intrarenal pressures. This concept is well explained in recent work by Jung et al., who analyzed the intrarenal pressures of 20 patients undergoing FURS, using an $8 \mathrm{ml} / \mathrm{min}$ irrigation system and a $20 \mathrm{ml}$ syringe as an additional irrigation system, they showed how intrarenal pressures, on average, reached $35( \pm 10)$ $\mathrm{mmHg}$ and how spikes higher than $288 \mathrm{mmHg}$ were not unusual using the syringe (9).

Our study also demonstrates how pump flow logically decreases with the use of higher caliber instruments that occupy the working channel of the ureteroscope.

The results are in concordance with our previous study evaluating the intrarenal pressure changes on a bench model with different instruments inside the ureteroscope (10).

However, the fact that there are no significant differences in flow between the pump on its own and the two fibers helps us understand how the system remains efficient particularly in the fragmentation phase and how, as a result, the flush should be reduced for clearer vision.

As there are no significant differences between the two fibers, the choice could switch to greater fiber size and greater power to break up the tougher stones.

Another advantage of using an automated irrigation system is connected to radiological exposure. In fact, during a standard FURS the operating urologist and the assistant that manages the manual watering system are both exposed to ionizing radiation. With an automated irrigation system instead, the operator is further away from the collimator seated in the console, and during the fragmentation phase when additional flow is often required, the assistant is not necessary since the additional flow is managed directly by the operator through the console.

\section{Conclusions}

The irrigation system of the new Avicenna Roboflex is optimized compared to the previous version, the flows developed with the ureteroscope and its various accessories appear to ensure adequate irrigation and a relatively acceptable pressure volume. However other more complex studies concerning the live flow/pressure relationship are needed before firm conclusions can be made.

\section{DisclosuRe}

Butticè S, Sahin B, Sener TE, Proietti S, Dragos L, Doizi S: nothing to disclose.

Traxer O: consultant for Coloplast, Rocamed, Olympus, Lumenis, Boston Scientific, Biohealth, EMS.

\section{References}

1. Rukin NJ, Siddiqui ZA, Chedgy EC, Somani BK. Trends in upper tract stone disease in England: evidence from the hospital episodes statistics database. Urol Int. 2017; 98:391-396.

2. Somani BK, Al-Qahtani SM, de Medina SDG, Traxer O. Outcomes of flexible ureterorenoscopy and laser fragmentation for renal stones: comparison between digital and conventional ureteroscope. Urology. 2013; 82:1017-9.

3. Saglam R, Muslumanoglu AY, Tokatli Z, et al. A new robot for flexible ureteroscopy: development and early clinical results (IDEAL stage 1-2b). Eur Urol. 2014; 66:1092-100.

4. Rassweiler J, Rassweiler MC, Klein J. New technology in ureteroscopy and percutaneous nephrolithotomy. Cur Opin Urol. 2016; 26:95-106.

5. Buttice S, Proietti S, Dragos L, Traxer O. Are you familiar with the flow of the Roboflex Avicenna pump? Allow me to explain. J Endourol. 2017; 31:418-419.

6. von Elm E, Altman DG, Egger M, et al. The strengthening the reporting of observational studies in epidemiology (STROBE) statement: guidelines for reporting observational studies. Int J Surg. 2014; 12:1495-9.

7. Bach T, Geavlete B, Herrmann T, Gross A. Working tools in flexible ureterorenoscopy -influence on flow and deflection: what does matter? J Endourol. 2008; 22:1639-44.

8. Seo H, Shin S, Jung N, et al. Scientific Program of 34th World Congress of Endourology \& SWL Program Book and Abstracts.J Endourol. 2016; 30 (S2):P1-A464.

9. Jung $H$, Osther PJ. Intraluminal pressure profiles during flexible ureterorenoscopy. Springerplus. 2015; 4:373

10. Sener TE, Cloutier J, Villa L, et al. Can we provide low intrarenal pressures with good irrigation flow by decreasing the size of ureteral access sheaths? J Endourol. 2016; 30:49-55.

\section{Correspondence}

Salvatore Butticè, $M D$

salvobu@gmail.com

Department of Urology, San Giovanni di Dio Hospital

Contrada Consolida, 92100, Agrigento, Italy

Bahadir Sahin, MD

drbahadirsahin@gmail.com

Tarik Emre Sener, MD

dr.emresener@gmail.com

Department of Urology, School of Medicine, Marmara University, Istanbul, Turkey

Laurian Dragos, MD

lauriandragos@yahoo.com

Department of Urology, Emergency County Hospital, Pius Branzeu, Timisoara, Romania

Silvia Proietti, MD

proiettisil@gmail.com

Ville Turro Division, Department of Urology, IRCCS, Ospedale San Raffaele, Milan, Italy

Steeve Doizi, MD

steeve.doizi@gmail.com

Olivier Traxer, MD

olivier.traxer@tnn.aphp.fr

Pierre \& Marie Curie University, Tenon University Hospital, Paris, France

PETRA UroGroup

Progress in Endourology, Technology and Research Association,

http://www.petraurogroup.org/ 